\title{
Associations of Elderly Onset Headache With Occurrence of Poor Functional Outcome, Cardiovascular Disease, and Cognitive Dysfunction During Long- term Follow-up
}

\author{
Soo-Jin $\mathrm{Cho}^{1}$, Byung-Kun Kim${ }^{2}$, Byung-Su Kim ${ }^{3}$, Jae-Moon Kim${ }^{4}$, Soo-Kyoung Kim ${ }^{5}$, Heui-Soo Moon ${ }^{6}$, Myoung-Jin Cha ${ }^{7}$, \\ Kwang-Yeol Park ${ }^{8}$, Jong-Hee Sohn ${ }^{9}$, Min Kyung Chu ${ }^{10}$, Tae-Jin Song ${ }^{11}$ \\ ${ }^{1}$ Department of Neurology, Dongtan Sacred Heart Hospital, Hallym University College of Medicine, Hwaseong, Korea \\ ${ }^{2}$ Department of Neurology, Eulji University School of Medicine, Seoul, Korea \\ ${ }^{3}$ Department of Neurology, Bundang Jesaeng General Hospital, Daejin Medical Center, Seongnam, Korea \\ ${ }^{4}$ Department of Neurology, Chungnam National University College of Medicine, Daejeon, Korea \\ ${ }^{5}$ Department of Neurology, Gyeongsang National University Hospital, Jinju, Korea \\ ${ }^{6}$ Department of Neurology, Kangbuk Samsung Hospital, Sungkyunkwan University School of Medicine, Seoul, Korea \\ ${ }^{7}$ Department of Neurology, National Police Hospital, Seoul, Korea \\ ${ }^{8}$ Department of Neurology, Chung-Ang University Hospital, Chung-Ang University College of Medicine, Seoul, Korea \\ ${ }^{9}$ Department of Neurology, Chuncheon Sacred Heart Hospital, Hallym University College of Medicine, Chuncheon, Korea \\ ${ }^{10}$ Department of Neurology, Severance Hospital, Yonsei University College of Medicine, Seoul, Korea \\ ${ }^{11}$ Department of Neurology, Ewha Womans University College of Medicine, Seoul, Korea
}

Corresponding Author:

Tae-Jin Song, MD, PhD

https://orcid.org/0000-0002-9937-762X

Department of Neurology, Ewha

Womans University College of

Medicine, 1071 Anyangcheon-ro,

Yangcheon-gu, Seoul 07985, Korea

Tel: +82-2-2650-2677

Fax: +82-2-2650-5958

E-mail: knstar@ewha.ac.kr

Received: August 20, 2018

Revised: October 8, 2018

Accepted: October 25, 2018
Background: Although the frequency and intensity of headaches decrease in older adults, headaches in this population are still an important neurological disorder. The purpose of this study was to investigate the associations of headache characteristics in older adults with the development of cardiovascular disease and cognitive dysfunction. Methods: We prospectively enrolled 125 older ( $\geq 65$ years old) patients with headache who were making their first visit to outpatient clinics and who had no prior history of cognitive dysfunction from 11 hospitals in Korea between August 2014 and February 2015. We investigated the occurrence of newly developed/or recurrent headache, cardiovascular disease, cognitive dysfunction, and poor functional outcomes. Results: The mean age of all included patients was 72.6 years, $68.8 \%$ were women, and 43 (34.4\%) had newly developed/or recurrent headache during follow-up. During a median follow-up of 31 months (interquartile range, 28-34 months), 21 participants (16.8\%) experienced cardiovascular disease, and 26 (20.8\%) developed cognitive dysfunction. Upon multivariate analysis and after adjusting for sex, age, and other factors, presence of newly developed/or recurrent headache was found to be associated with cardiovascular disease (hazard ratio [HR], 4.03; 95\% confidence interval [Cl], 1.28-12.61; $p=0.017$ ) and frequency of headache for the recent 3 months was related with cognitive dysfunction (HR, 1.05; 95\% Cl, 1.00-1.09; $p=0.017$ ) and poor functional outcomes (HR, 1.06; 95\% Cl, 1.01-1.11; $p=0.011)$. Conclusion: Our study demonstrated that there is an increased risk of cardiovascular disease, cognitive dysfunction, and poor functional outcomes in older patients with frequent, newly developed, or recurrent headache. (Ann Geriatr Med Res 2018;22:176-183)

Key Words: Migraine disorders, Tension-type headache, Elderly headache, Cardiovascular diseases, Cognitive dysfunction

\section{INTRODUCTION}

Headache is one of the most frequent neurological problems that reported in nearly half of older adults. ${ }^{1,2)}$ The characteristics of headache in older adults are different from those reported by younger patients. ${ }^{3)}$ Older patients tend to experience more migraine characteristics, hypnic headaches, facial neuralgia, and secondary headaches, which may be related with aging (metabolic or hormonal changes, comorbidities, or prescribed medications). ${ }^{3)}$ Accordingly, a prudential investigation into the characteristics and prognosis of headache is mandatory for the appropriate management and treatment of older patients with headache.

Few studies have investigated the association between headache disorders and long-term prognosis. In the case 
of migraine, a major primary headache disorder, there was an association with cardiovascular disease events, including cardiovascular mortality, in young to middle-aged women. ${ }^{4)}$ However, detailed information and the longterm prognosis of headache in older patients, especially on new onset headache, have been rarely reported. Previous studies on the prevalence of headache have focused on this condition only in relatively younger patients, with a limited study sample size of older participants; the International Classification of Headache Disorders, Third Edition (ICHD-3) and ICHD-3 beta version have also not been used. $^{5-8)}$ Moreover, one other study that carried out longterm follow-up for headaches in older adults only included a small number of patients with chronic daily headache. ${ }^{9)}$

Therefore, the purpose of this study was (1) to investigate the characteristics of headache of older patients; (2) to check whether the headache in older patients improved, recurred, worsened, or became chronic; and (3) to confirm the association of headache in older patients with the occurrence of poor functional outcomes, cardiovascular diseases, and cognitive dysfunction during long-term followup by using a multicenter headache registry data set.

\section{MATERIALS AND METHODS}

\section{Participants}

This study was performed as a sub-study of a prospective, multicenter headache registry called the "headache registry using the ICHD-3 beta version for first-visit patients" (HEREIN). ${ }^{10,11)}$ The data set of the HEREIN study was prospectively acquired from patients visiting outpatient headache clinics for the first time; patients visiting neurology departments of 11 hospitals (6 tertiary and 5 secondary referral hospitals) in Korea between August 2014 and February 2015 were enrolled. ${ }^{12)}$ We reviewed the overall headache diagnosis at baseline and/or follow-up (if presented) and determined the type using the ICHD-3 beta version criteria. Participants who listed headache as the chief complaint for the outpatient clinic visit were all Korean adults (from 19-100 years of age) without any difficulty in communication or conversation that would prohibit accurate history taking. Patients with impaired cognitive function, aphasia/severe dysarthria, or any other serious medical or psychiatric condition, identified based on a physician's evaluation, were excluded, as were those who visited the hospital for major symptoms other than headache. ${ }^{10)}$ The Institutional Review Board of each hospital approved this study. All patients provided informed consent for participation in our study.

\section{Baseline Headache Assessment and Diagnosis}

All included patients completed a self-administered survey and were examined by a headache specialist. In cases where secondary headaches had to be excluded, blood, laboratory, and/or cerebrospinal fluid examinations were performed. Brain imaging (computed tomography [CT] and/or magnetic resonance imaging [MRI]) including the cerebral vasculature was also performed based on the headache specialist's judgment at each center. The survey and questionnaire assessed the patients' history and characteristics of their headaches, including presence of aura, severity of headache (visual analogue scale [VAS]), pain characteristics, location, headache attack duration, presence of aggravation with routine physical activity, any accompanying symptoms, and autonomic symptoms or features such as vertigo. ${ }^{12)}$ The confirmative headache diagnosis was performed based on the ICHD- $3 \beta$ criteria after considering the questionnaire results, medical records, neurological examination, laboratory findings, neuroimaging studies, and treatment response. Controversial cases were reviewed by researchers through case analysis meetings until a consensus was reached. The interrater agreement among the researchers, calculated using Fleiss' kappa coefficients, was $0.61 .^{10,13)}$

\section{Design of the Follow-up Study and Definitions of Clinical Outcomes}

To perform our prospective observational study, we utilized the data set from a previous study, which investigated the characteristics of 152 older patients with headache. ${ }^{12)}$ Out of these 152 patients, we excluded those who did not want to participate in our follow-up study and who were not contacted $(n=27)$; the remaining 125 patients agreed to provide clinical information for followup. These 125 participants were followed up from April 2015 to April 2018. A board-certified headache specialist obtained the participants' clinical information at the out-patient clinic during face-to-face and/or telephone interviews. When patients did not revisit during scheduled follow-ups, their clinical information was acquired via a telephone interview with the patient and/or a close relative. The definition of "newly developed headache" was a newly developed headache as the chief complaint following complete remission of the index headache. A recurrent headache was defined as the recurrence of the initial headache without definite remission.

At the follow-up time, we acquired clinical information on frequency of headache for the most recent 3 months following the last follow-up regarding (1) headache recurrence, continuation, and/or changes in characteristics and (2) occurrence of cardiovascular disease, cognitive dysfunction, and change in functional status, including mortality (modified Rankin scale [mRS]). The primary outcome was major adverse cardiovascular events (sum of the occurrence of all-cause mortality, unstable angina, myocardial infarction, coronary revascularization, and stroke). ${ }^{14)}$ Cognitive dysfunction was defined as diagnosis of mild cognitive impairment (MCI) or dementia based on previous studies. ${ }^{15)}$ The development and type of cognitive dysfunction were confirmed at each hospital by a neurology specialist. The mRS is commonly used for assessing functional outcomes (0, no symptoms; 1 , no significant disability; 2 , 
slight disability; able to look after own affairs without assistance, but unable to carry out all previous activities; 3, moderate disability; requires some help, but able to walk unassisted; 4, moderately severe disability; unable to attend to own bodily needs without assistance, and unable to walk unassisted; 5, severe disability; requires constant nursing care and attention, bedridden, incontinent; and 6, dead) for measuring the degree of disability or dependence in the daily activities of people. This follow-up study protocol was reviewed and approved by the ethics committee of each hospital. Based on the decision of the Institutional Review Board of each hospital, our data was acquired after receiving informed consent or oral agreements by telephone interview from participants and/or their caregiver.

\section{Statistical Analysis}

Statistical analyses were performed using the IBM SPSS Statistics ver. 21.0 (IBM Co., Armonk, NY, USA). Continuous variables are presented as means \pm standard deviations. Categorical variables are shown as frequencies and percentages. Continuous or interval-scaled variables were compared using Student t-test or the Mann-Whitney Utest, while all categorical variables were compared using the chi-square test or Fisher exact test. Due to the small sample size, patients were dichotomized based upon clinical outcomes, poor functional status ( $m R S \geq 2$ or $<2$ ), cognitive dysfunction (occurrence of MCI and/or dementia vs. no occurrence), and whether newly developed headache and/or recurrent headache existed; the associations of the index headache characteristics with clinical outcomes were then analyzed using univariate analysis (KaplanMeier product-limit method with a log-rank test) and multivariate Cox proportional hazards regression. In addition to age and sex, variables with $\mathrm{p}<0.1$ upon univariate analysis were entered into the multivariate analyses. A 2-tailed $\mathrm{p}<0.05$ was considered statistically significant. There was no statistical interaction between frequency of headache for the most recent 3 months following the last follow-up and newly developed headache and/or recurrent headache with the occurrence of poor functional outcomes ( $p$ for interaction=0.999) or cardiovascular disease ( $p$ for interaction $=0.724$ ) or cognitive dysfunction ( $p$ for interaction=0.819).

\section{RESULTS}

\section{Demographic Data of the Study Population}

The age of the 125 patients was $72.6 \pm 5.9$ years (mean \pm standard deviation), and there were more women (86 women [68.8\%] vs. 39 men [31.2\%]) in the study. Brain CT was performed in 33 participants (26.4\%), and brain MRI was carried out in 53 (42.4\%). The most common type of headache was primary headache $(n=103$ [82.4\%]), followed by secondary headache $(n=14[11.2 \%])$, painful cranial neuropathies, and other facial pains $(n=8$ [6.4\%]). Among the primary headaches, tension-type headaches $(n=57$
[45.6\%]) and other primary headaches $(n=35$ [28.0\%]), particularly primary stabbing headaches ( $n=22$ [17.6\%]), were most frequently noted. Considering secondary headache, infection ( $n=5[4.0 \%])$, facial pain attributed to disorder of the cranium $(n=4[3.2 \%])$, and cranial vascular disorders $(\mathrm{n}=2[1.6 \%])$ were most frequently noted. Medication overuse headache was noted in nine (7.2\%) participants.

Among the 125 patients, 43 (34.4\%) had newly developed headache and/or recurrent headache; in addition, tension-type headache $(n=23$ [53.5\%]), other primary headache $(n=12$ [27.9\%]), migraine $(n=5$ [11.6\%], trauma related $(n=1[2.3 \%])$, cranial vascular disorder $(n=1[2.3 \%])$, and facial pain attributed to disorder of the cranium cranial $(n=1$ [2.3\%]) were also commonly noted. In 12 other primary headache patients, primary stabbing headache was most frequent $(n=8[66.7 \%])$. Considering newly developed headache and/or recurrent headache, the three patients with migraine reported another type of headache (tension-type headache) as well, and 8 patients with tension-type headache also experienced another type of headache (migraine). Compared with the no newly developed headache and/or recurrent headache group, those who reported newly developed headache and/or recurrent headache had a higher frequency of headache for the most recent 3 months following the last follow-up $(9.5 \pm 9.8$ days vs. $1.5 \pm 1.0$ day, $p=0.001$ ) and primary headache disorders $(\mathrm{p}=0.078)$ (Table 1).

\section{Associations of Headache Characteristics With Clinical Outcomes}

During the median 31 months (interquartile range: 28-34 months) of follow-up, most patients reported an improvement in their index headache $(n=106$ [84.8\%]), in contrast, $15(12.0 \%)$ patients complained of no significant change of index headache and 4 (3.2\%) patients described their headache as progressively worse following the last followup. Two patients (1.2\%) (1 had chronic migraine and 1 had probable tension-type headache) had chronic daily headache (1 had chronic migraine and 1 had chronic tensiontype headache).

Considering clinical outcomes, 23 patients (18.4\%) had a poor functional outcome, 21 (16.8\%) developed cardiovascular disease, and 26 (20.8\%) reported cognitive dysfunction. Kaplan-Meier curves demonstrated that poor functional outcomes $(p=0.014)$, cardiovascular disease $(p=0.009)$, and cognitive dysfunction $(p=0.009)$ depended on the presence of newly developed headache and/or recurrent headache (Fig. 1). Upon multivariate analysis and after adjusting for sex, age, and other factors that were at least marginally significant in univariate analysis $(\mathrm{p}<0.1)$ (Table 2), high frequency of headache for the most recent 3 months following the last follow-up was related with poor functional outcomes (hazard ratio [HR], 1.06; 95\% confidence interval [CI], 1.01-1.11; $\mathrm{p}=0.011)$ and the occurrence of cognitive dysfunction (HR, 1.05; 95\% CI, 1.01-1.09; $p=0.017)$, but not with the occurrence of car- 
Table 1. Demographics and characteristics of older patients with headache according to the presence of newly developed headache and/or recurrent headache

\begin{tabular}{|c|c|c|c|c|}
\hline Variable & Total $(n=125)$ & $\begin{array}{l}\text { No newly developed } \\
\text { headache and/or } \\
\text { recurrent headache } \\
(n=82)\end{array}$ & $\begin{array}{l}\text { Newly developed } \\
\text { headache and/or } \\
\text { recurrent headache } \\
(\mathrm{n}=43)\end{array}$ & p-value \\
\hline \multicolumn{5}{|l|}{ Demographic data } \\
\hline Female sex & $86(68.8)$ & $55(67.1)$ & $31(72.1)$ & 0.565 \\
\hline Age (yr) & $72.6 \pm 5.9$ & $72.4 \pm 5.8$ & $72.8 \pm 6.3$ & 0.726 \\
\hline Age of headache onset (yr) & $71.5 \pm 5.9$ & $71.5 \pm 5.7$ & $71.6 \pm 6.3$ & 0.943 \\
\hline Types of brain imaging performed & & & & 0.517 \\
\hline Brain CT & $33(26.4)$ & $22(26.8)$ & $11(25.6)$ & \\
\hline Brain MRI & $53(42.4)$ & $32(39.0)$ & $21(48.8)$ & \\
\hline Visual analogue scale & $5.4 \pm 1.9$ & $5.6 \pm 1.9$ & $5.2 \pm 1.8$ & 0.192 \\
\hline Duration of index headache (day) per month & $6.8 \pm 20.5$ & $6.1 \pm 21.3$ & $8.2 \pm 19.2$ & 0.589 \\
\hline Types of headache or cranial neuralgia & & & & 0.078 \\
\hline Primary headache & $103(82.4)$ & $63(76.8)$ & $40(93.0)$ & \\
\hline Secondary headache & $14(11.2)$ & $12(14.6)$ & $2(4.7)$ & \\
\hline Painful cranial neuropathies and other facial pains & $8(6.4)$ & $7(8.5)$ & $1(2.3)$ & \\
\hline \multicolumn{5}{|l|}{ Subtypes of headache } \\
\hline Primary headache & & & & 0.755 \\
\hline Migraine & $11(8.8)$ & $6(7.3)$ & $5(11.6)$ & \\
\hline Tension type headache & $57(45.6)$ & $34(41.5)$ & $23(53.5)$ & \\
\hline Other primary headache & $35(28.0)$ & $23(28.0)$ & $12(27.9)$ & \\
\hline Secondary headache & & & & 0.163 \\
\hline Trauma related & $1(0.8)$ & $0(0)$ & $1(2.3)$ & \\
\hline Cranial vascular disorder & $2(1.6)$ & $2(2.4)$ & $0(0)$ & \\
\hline Substance & $1(0.8)$ & $1(1.2)$ & $0(0)$ & \\
\hline Infection & $5(4.0)$ & $5(6.1)$ & $0(0)$ & \\
\hline Homeostasis & $1(0.8)$ & $1(1.2)$ & $0(0)$ & \\
\hline Facial pain attributed to disorder of the cranium & $4(3.2)$ & $3(3.7)$ & $1(2.3)$ & \\
\hline Painful cranial neuropathies and other facial pains & & & & 0.375 \\
\hline Neuralgia & $3(2.4)$ & $2(2.4)$ & $1(2.3)$ & \\
\hline Other headache disorders & $5(4.0)$ & $5(6.1)$ & $0(0)$ & \\
\hline Medication overuse headache & $9(7.2)$ & $4(4.9)$ & $5(11.6)$ & 0.273 \\
\hline Kinds of medications for medication overuse headache & & & & 0.538 \\
\hline Triptans & $3(2.4)$ & $1(1.2)$ & $2(4.7)$ & \\
\hline NSAID & $4(3.2)$ & $2(2.4)$ & $2(4.7)$ & \\
\hline Combined pain killers & $1(0.8)$ & $1(1.2)$ & $1(2.3)$ & \\
\hline Frequency of headache for recent 3 months (day) & $3.2 \pm 7.3$ & $1.5 \pm 1.0$ & $9.5 \pm 9.8$ & 0.001 \\
\hline
\end{tabular}

Values are presented as number (\%) or mean \pm standard deviation.

CT, computed tomography; MRI, magnetic resonance image; NSAID, nonsteroidal anti-inflammatory drug.

A

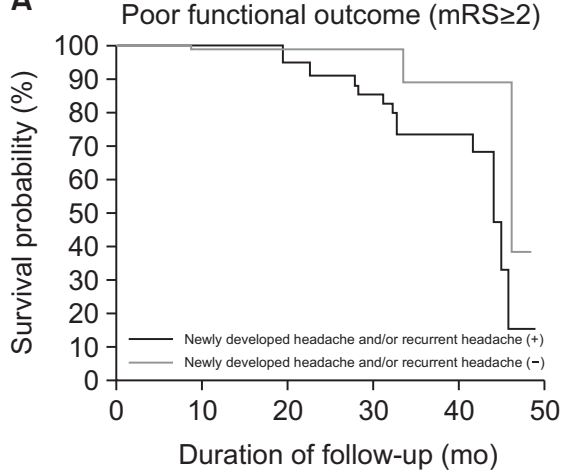

B Occurence of cardiovascular disease $\mathrm{C}$

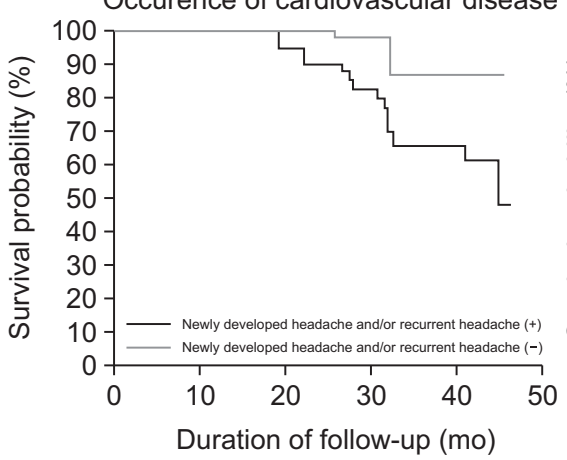

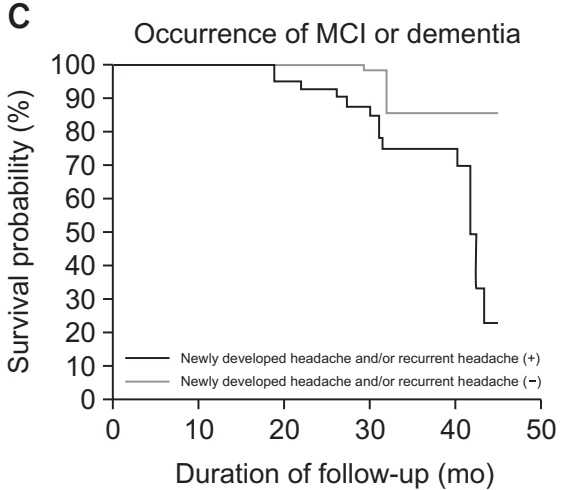

Fig. 1. Kaplan-Meier survival plots of long-term, poor functional outcomes (A), occurrence of cardiovascular disease (B), and incidence of cognitive dysfunction (C) in the presence of newly developed headache and/or recurrent headache in older adults. Duration of follow-up was noted as months. mRS, modified Rankin scale; MCI, mild cognitive impairment. 
Table 2. Univariate analysis for association of characteristics of older patients with headache

\begin{tabular}{|c|c|c|c|}
\hline Variable & Cardiovascular disease & Cognitive dysfunction & Poor functional outcomes \\
\hline \multicolumn{4}{|l|}{ Demographic data } \\
\hline Female sex & $0.68(0.25-1.88)$ & $0.37(0.12-1.08)$ & $0.98(0.38-2.51)$ \\
\hline Age & 0.99 (0.93-1.06) & $1.02(1.01-1.04)^{*}$ & $1.00(0.95-1.06)$ \\
\hline Age of headache onset & $0.98(0.91-1.05)$ & $1.01(0.99-1.02)$ & $0.99(0.93-1.05)$ \\
\hline \multicolumn{4}{|l|}{ Types of brain imaging performed } \\
\hline No brain imaging performed & Reference & Reference & Reference \\
\hline Brain CT & $1.09(0.34-3.48)$ & $2.47(0.87-6.99)$ & $1.84(0.65-5.19)$ \\
\hline Brain MRI & $0.86(0.32-2.31)$ & $1.19(0.44-3.23)$ & $0.62(0.23-1.68)$ \\
\hline Visual analog scale & $1.15(0.94-1.42)^{\dagger}$ & $0.97(0.79-1.18)$ & $1.03(0.82-1.27)$ \\
\hline Duration of index headache (day) per month & $0.99(0.96-1.03)$ & $1.00(0.97-1.02)$ & $0.99(0.95-1.03)$ \\
\hline \multicolumn{4}{|l|}{ Types of headache or cranial neuralgia } \\
\hline Primary headache & $0.67(0.24-1.85)$ & $1.47(0.44-4.94)$ & $0.72(0.22-3.28)$ \\
\hline Secondary headache & $1.95(0.65-5.87)$ & $1.32(0.45-2.42)$ & $2.28(0.75-7.70)$ \\
\hline Painful cranial neuropathies and other facial pains & $0.69(0.09-5.25)$ & $1.51(0.35-6.59)$ & $0.57(0.45-14.29)$ \\
\hline Medication overuse headache & $2.26(0.73-6.93)$ & $1.12(0.37-3.38)$ & $1.29(0.42-3.98)$ \\
\hline Frequency of headache for recent 3 months (day) & $1.02(0.97-1.07)^{\dagger}$ & $1.05(1.01-1.09)^{*}$ & $1.06(1.02-1.11)^{*}$ \\
\hline Newly developed headache and/or recurrent headache & $2.67(1.05-6.82)^{*}$ & $2.59(1.04-6.43)^{*}$ & $3.08(1.09-8.71)^{*}$ \\
\hline
\end{tabular}

Values are presented as hazard ratio (95\% confidence interval).

$\mathrm{CT}$, computed tomography; MRI, magnetic resonance image.

${ }^{*} \mathrm{p}<0.05 .{ }^{\dagger} \mathrm{p}<0.1$.

Table 3. Multivariate analysis for association of characteristics of older patients with headache

\begin{tabular}{lccc}
\hline \multicolumn{1}{c}{ Variable } & Cardiovascular disease & Cognitive dysfunction & Poor functional outcomes \\
\hline Female sex & $0.85(0.30-2.35)$ & $0.37(0.12-1.08)^{\dagger}$ & $0.73(0.29-1.87)$ \\
Age & $0.99(0.92-1.06)$ & $0.98(0.91-1.05)$ & $1.02(0.97-1.08)$ \\
Visual analog scale & $1.25(1.01-1.56)^{*}$ & - & - \\
Frequency of headache for recent 3 months (day) & $0.99(0.93-1.05)$ & $1.05(1.01-1.09)^{*}$ & $1.06(1.01-1.11)^{*}$ \\
Newly developed headache and/or recurrent headache & $4.03(1.28-12.61)^{*}$ & $1.53(0.50-1.53)$ & $1.86(0.55-6.32)$ \\
\hline
\end{tabular}

Values are presented as hazard ratio (95\% confidence interval).

${ }^{*} \mathrm{p}<0.05 .{ }^{\dagger} \mathrm{p}<0.1$.

diovascular disease (HR, 0.99; 95\% CI, 0.93-1.05; $\mathrm{p}=0.905)$ (Table 3). The presence of newly developed headache and/ or recurrent headache was significantly associated with the occurrence of cardiovascular disease (HR, 4.03; 95\% CI, 1.28-12.61; $\mathrm{p}=0.017$ ) but not with poor functional outcomes (HR, 1.86; 95\% CI, 0.55-6.32; $\mathrm{p}=0.315)$ and cognitive dysfunction (HR, 1.53; 95\% CI, 0.50-1.53; $\mathrm{p}=0.448$ ) (Table 3).

\section{DISCUSSION}

The key findings from our prospective, first-visit, outpatient-based, multicenter study were as follows: (1) even though most headaches improved, 43 patients (34.4\%) had newly developed/or recurrent headache during follow-up, and tension-type and other primary headaches (mainly primary stabbing headache) were the most commonly reported newly developed/recurrent headaches in older adults; (2) the occurrence of cardiovascular disease was related with newly developed headache and/or recurrent headache in older adults; and (3) a high frequency of headache for the most recent 3 months following the last follow-up also showed an association with the development of cognitive dysfunction and poor functional outcomes.

In our study, tension-type headache was the most common headache reported by older patients. Previous studies have found the prevalence of older adults with tension headache to range from $16 \%-44 \% .{ }^{16-19)}$ Moreover, one clinic-based study demonstrated that the frequency of tension headache in older adults was approximately 30\%. Our results are similar to those of these previous studies and provide additional information regarding older Korean patients with headache. In addition, in our dataset, the second most frequent primary headache disorder was other primary headache, especially primary stabbing headache. These results are similar to the findings of a previous study ${ }^{20)}$ but different from the results gathered using a relatively younger-onset headache population. ${ }^{2)}$ These discrepancies may be explained by hormonal variations, underlying vascular risk factors, concomitant medications, and decreased physical activity in older adults. ${ }^{21)}$ The precise reason why primary stabbing headache is commonly noted in older patients with headache is unknown; 
however, some possible explanations exist. First, tortuosity of the cerebral vasculature and increased frequency of cervicogenic headache and neuralgia are commonly noted in older adults; these factors may affect the occurrence of primary stabbing headache. ${ }^{22,23)}$ Second, in our study, a diagnosis of headache was made based on the ICHD-3 $\beta$ (initial) and ICHD-3 (follow-up). The diagnostic yield for other primary headaches using the ICHD- $3 \beta$ and ICHD- 3 is more favorable than that of the ICHD-2. ${ }^{8,11}$ Third, because the diagnostic criteria in the ICHD- $3 \beta$ and ICHD-3 for primary stabbing headache for the first division of trigeminal nerve distribution were removed, the possibility of a diagnosis of primary stabbing headache was increased. ${ }^{8,11)}$

As expected, in our study, the frequency of headache for the most recent 3 months following the last follow-up was related with newly developed headache and/or recurrent headache. As the frequency of headache increases, peripheral and central sensitization occurs, and headache thresholds change, leading to headache chronification. ${ }^{24)}$ In fact, previous studies have shown that the likelihood of subsequent chronification increases with the frequency of migraine and tension-type headache. ${ }^{25,26)}$ Our study has significant implications for providing evidence of headache chronification, particularly in older patients, who typically report relatively fewer headaches than younger people.

In our study of older patients with headache, presence of newly developed headache and/or recurrent headache was associated with long-term cardiovascular disease occurrence. In previous studies, migraine was a risk factor for recurrent ischemic stroke and vascular events. ${ }^{27)}$ In contrast, another population-based study reported that the association of migraine with ischemic stroke was significantly related with a lower Framingham risk score. ${ }^{28)}$ A Northern Manhattan study of 1,292 migraine patients found that the risk of stroke was increased in older (mean age, 68 years) migraineurs. ${ }^{29)}$ The increased risk of cardiovascular disease, including stroke, in migraine patients has been suggested to be due to concomitant vascular risk factors or genetic components. ${ }^{30)}$ However, in our dataset, the frequency of tension-type headache and other primary headache was higher than that of migraine. In a general population-based study conducted in Taiwan, tension-type headache patients showed 2 fold higher risk of ischemic stroke compared to those who without tension-type headache. ${ }^{31)}$ Thus, although our study cannot provide detailed mechanisms, our data on older patients with headache remains significant as it provided additional information regarding the association of newly developed headache and/ or recurrent headache in older patients with cardiovascular disease occurrence during long-term follow-up.

The risk of dementia was found to be increased in a nationwide cohort study, especially in individuals with tension-type headaches. ${ }^{32)}$ Another previous study showed that tension-type headaches were associated with impaired cognitive function. ${ }^{33)}$ It is interesting to note that migraineurs, particularly migraineurs with aura, tended to score higher on cognition tests than nonmigraineurs in the Rotterdam Study. ${ }^{34)}$ In contrast, chronic headache or frequent headache in older patients was related with a higher incidence of seeking care for cognitive decline. ${ }^{35}$ Our findings, which included relatively more patients with tension-type headache, are in line with the results of previous studies and may reconfirm the increased risk of cognitive decline in older patients who have a high frequency of headache or who have newly developed headache and/ or recurrent headache (even though this was significant in the univariate analysis). Although a clear mechanism has not been identified, there are hypotheses that can explain the link between headaches and cognitive dysfunction. First, the brain structures involved in pain processing related to headache, such as the cingulate gyrus, prefrontal cortex, and thalamus, are also closely related to dementia and cognitive dysfunction. ${ }^{36)}$ That is, if the neural network is damaged by persistent pain, cognition impairment is likely to occur. ${ }^{37)}$ Second, patients with migrane have relatively high vascular risk factors, which may result in vascular dementia or vascular cognitive dysfunction. ${ }^{4)} \mathrm{Fi}$ nally, neuropsychological disorders that are uncommon in patients with headache might be important risk factors for cognitive decline. ${ }^{38)}$ Further research on the mechanism of this association is warranted

Although studies on headaches in older adults and its functional outcomes have rarely been conducted and are difficult to compare with previous studies, our study showed that a recent high frequency of headaches in this population was associated with long-term poor functional outcomes. The reason for this association is likely that frequent headaches might be linked with increased disability due to the headaches per se or a greater risk of cardiovascular disease, especially stroke. However, since we cannot examine the baseline mRS, and our sample size was small, further studies are needed to confirm these hypotheses.

Our study had some limitations. First, only a small sample size of older patients was included in the study. Therefore, subgroup analysis could not be performed. Second, this study does not represent the general population, since our study was a hospital-based long-term follow-up study. Third, our research could not explain the exact mechanism of the association with cardiovascular disease and cognitive dysfunction. Fourth, our study did not acquire objective baseline information for cognitive function such as the mini-mental state examination, educational level, and neuropsychological disorders (anxiety or depression), which are important factors affecting future cognitive function. Finally, because our original dataset consisted of epidemiological information concerning headaches in older adults, we could not acquire data concerning the presence of vascular risk factors, medical histories, medication history, and medication compliance either at baseline or during follow-up. Despite these limitations, our study has the following strengths. First, our study confirmed the 
long-term prognosis of older patients with headache, and the findings demonstrate the different factors associated with prognosis; these factors are relatively uncommon in young or middle-aged patients. ${ }^{28,32)}$ Second, our study was aimed at older Asian patients, unlike previous studies on Western patients. ${ }^{1921)}$ Third, the prognosis of older patients with headache was confirmed using the ICHD-3 version, which was recently updated. ${ }^{19)}$ Finally, unlike previous studies which demonstrated the association of headaches in older people with one outcome parameter, ${ }^{31-34)}$ our study comprehensively investigated important outcomes, such as cardiovascular disease, cognitive dysfunction, and functional outcomes.

In conclusion, our study demonstrated that there is a risk of cardiovascular disease, cognitive dysfunction, and poor functional outcomes in older patients with a recently high frequency of headache or presence of newly developed headache and/or recurrent headache.

\section{CONFLICTS OF INTEREST DISCLOSURES}

The researchers claim no conflicts of interest.

\section{ACKNOWLEDGMENTS}

This study was supported by a 2016 junior researcher grant from the Korean Geriatric Society.

\section{REFERENCES}

1. Hale WE, Perkins LL, May FE, Marks RG, Stewart RB. Symptom prevalence in the elderly. An evaluation of age, sex, disease, and medication use. J Am Geriatr Soc 1986;34:333-40.

2. Ruiz M, Pedraza MI, de la Cruz C, Barón J, Muñoz I, Rodríguez C, et al. Headache in the elderly: characteristics in a series of 262 patients. Neurologia 2014;29:321-6.

3. Bamford CC, Mays M, Tepper SJ. Unusual headaches in the elderly. Curr Pain Headache Rep 2011;15:295-301.

4. Kurth T, Winter AC, Eliassen AH, Dushkes R, Mukamal KJ, Rimm $\mathrm{EB}$, et al. Migraine and risk of cardiovascular disease in women: prospective cohort study. BMJ 2016;353:i2610.

5. Kim BK, Chu MK, Lee TG, Kim JM, Chung CS, Lee KS. Prevalence and impact of migraine and tension-type headache in Korea. J Clin Neurol 2012;8:204-11.

6. Peng KP, Wang SJ. Epidemiology of headache disorders in the Asia-pacific region. Headache 2014;54:610-8.

7. Pryse-Phillips W, Findlay H, Tugwell P, Edmeads J, Murray TJ, Nelson RF. A Canadian population survey on the clinical, epidemiologic and societal impact of migraine and tension-type headache. Can J Neurol Sci 1992;19:333-9.

8. Headache Classification Committee of the International Headache Society (IHS) The International Classification of Headache Disorders, 3rd edition. Cephalalgia 2018;38:1-211.

9. Fuh JL, Wang SJ, Lu SR, Tsai PH, Lai TH, Lai KL. A 13-year longterm outcome study of elderly with chronic daily headache. Cephalalgia 2008;28:1017-22.
10. Cho SJ, Kim BK, Kim BS, Kim JM, Kim SK, Moon HS, et al. Vestibular migraine in multicenter neurology clinics according to the appendix criteria in the third beta edition of the International Classification of Headache Disorders. Cephalalgia 2016;36:454-62.

11. Headache Classification Committee of the International Headache Society (IHS). The International Classification of Headache Disorders, 3rd edition (beta version). Cephalalgia 2013;33:629-808.

12. Song TJ, Kim YJ, Kim BK, Kim BS, Kim JM, Kim SK, et al. Characteristics of elderly-onset ( $\geq 65$ years) headache diagnosed using the International Classification of Headache Disorders, Third Edition Beta Version. J Clin Neurol 2016;12:419-25.

13. Cha MJ, Moon HS, Sohn JH, Kim BS, Song TJ, Kim JM, et al. Chronic daily headache and medication overuse headache in firstvisit headache patients in Korea: a multicenter clinic-based study. J Clin Neurol 2016;12:316-22.

14. Yoo J, Song D, Baek JH, Kim K, Kim J, Song TJ, et al. Poor longterm outcomes in stroke patients with asymptomatic coronary artery disease in heart CT. Atherosclerosis 2017;265:7-13.

15. Hong YJ, Yang DW, Yoon B, Shim YS, Cho AH, Han IW, et al. One-year follow-up pilot study for dementia diagnosis in elderly with recall score of 0 in Korean-version of Mini-Mental State Examination. J Korean Geriatr Soc 2014;18:16-23.

16. Kaniecki RG. Tension-type headache in the elderly. Curr Treat Options Neurol 2007;9:31-7.

17. Schwaiger J, Kiechl S, Seppi K, Sawires M, Stockner H, Erlacher T, et al. Prevalence of primary headaches and cranial neuralgias in men and women aged 55-94 years (Bruneck Study). Cephalalgia 2009;29:179-87.

18. Crystal SC, Grosberg BM. Tension-type headache in the elderly. Curr Pain Headache Rep 2009;13:474-8.

19. Pérez-Martínez D, Puente-Muñoz A, Anciones B. Headache among oldest old ( +75 years): findings from 736 consecutive subjects in outpatient neurological clinic. Neurologia 2008;23:436-40.

20. Fuh JL, Kuo KH, Wang SJ. Primary stabbing headache in a headache clinic. Cephalalgia 2007;27:1005-9.

21. Prencipe M, Casini AR, Ferretti C, Santini M, Pezzella F, Scaldaferri $\mathrm{N}$, et al. Prevalence of headache in an elderly population: attack frequency, disability, and use of medication. J Neurol Neurosurg Psychiatry 2001;70:377-81.

22. Edmeads J. The cervical spine and headache. Neurology 1988;38:1874-8.

23. Love $S$, Coakham HB. Trigeminal neuralgia: pathology and pathogenesis. Brain 2001;124(Pt 12):2347-60.

24. Pak DJ, Yong RJ, Kaye AD, Urman RD. Chronification of pain: mechanisms, current understanding, and clinical implications. Curr Pain Headache Rep 2018;22:9.

25. May A, Schulte LH. Chronic migraine: risk factors, mechanisms and treatment. Nat Rev Neurol 2016;12:455-64.

26. Bezov D, Ashina S, Jensen R, Bendtsen L. Pain perception studies in tension-type headache. Headache 2011;51:262-71.

27. Pezzini A, Grassi M, Lodigiani C, Patella R, Gandolfo C, Zini A, et al. Predictors of long-term recurrent vascular events after ischemic stroke at young age: the Italian Project on Stroke in Young Adults. Circulation 2014;129:1668-76.

28. Kurth T, Schürks M, Logroscino G, Gaziano JM, Buring JE. Migraine, vascular risk, and cardiovascular events in women: pro- 
spective cohort study. BMJ 2008;337:a636.

29. Monteith TS, Gardener H, Rundek T, Elkind MS, Sacco RL. Migraine and risk of stroke in older adults: Northern Manhattan Study. Neurology 2015;85:715-21.

30. Lee MJ, Lee C, Chung CS. The migraine-stroke connection. J Stroke 2016;18:146-56.

31. Tsai CL, Chou CH, Lee PJ, Yin JH, Chen SY, Lin CC, et al. The potential impact of primary headache disorders on stroke risk. J Headache Pain 2016;17:108.

32. Yang FC, Lin TY, Chen HJ, Lee JT, Lin CC, Kao CH. Increased risk of dementia in patients with tension-type headache: a nationwide retrospective population-based cohort study. PLoS One 2016;11:e0156097.

33. Smith AP. Acute tension-type headaches are associated with impaired cognitive function and more negative mood. Front Neurol 2016;7:42.
34. Wen K, Nguyen NT, Hofman A, Ikram MA, Franco OH. Migraine is associated with better cognition in the middle-aged and elderly: the Rotterdam Study. Eur J Neurol 2016;23:1510-6.

35. Feleppa M, Fucci S, Bigal ME. Primary headaches in an elderly population seeking medical care for cognitive decline. Headache 2017;57:209-16.

36. Apkarian AV, Bushnell MC, Treede RD, Zubieta JK. Human brain mechanisms of pain perception and regulation in health and disease. Eur J Pain 2005;9:463-84.

37. Svoboda E, McKinnon MC, Levine B. The functional neuroanatomy of autobiographical memory: a meta-analysis. Neuropsychologia 2006;44:2189-208.

38. Johansson L, Guo X, Waern M, Ostling S, Gustafson D, Bengtsson C, et al. Midlife psychological stress and risk of dementia: a 35year longitudinal population study. Brain 2010;133(Pt 8):2217-24. 